SMASIS2014-7565

\title{
MODELING OF A HEAVING BUOY WAVE ENERGY CONVERTER WITH STACKED DIELECTRIC ELASTOMER GENERATOR
}

\author{
Giacomo Moretti \\ Marco Fontana \\ Rocco Vertechy \\ PERCRO SEES, TeCIP Institute, Scuola Superiore Sant'Anna \\ Piazza Martiri della Libertà 33, Pisa, 5612, Italy \\ E-mail: m.fontana@sssup.it, r.vertechy@sssup.it
}

\begin{abstract}
This paper introduces a novel architecture of Wave Energy Converter (WEC) provided with a Dielectric Elastomer (DE) Power Take-Off (PTO) system. The device, named Poly-Buoy, includes a heaving buoy as primary interface, that captures the mechanical energy from waves, and a DE Generator (DEG), made by stacked layers of silicone elastomer, that converts mechanical energy into electricity.

A mathematical model of the Poly-Buoy is proposed, which includes analytical electro-hyperlastic equations for the DEG and a linear model for wave-buoy hydrodynamics.

Procedures for the design and optimization of different layouts and control strategies for the DE-PTO are introduced that specifically consider single-DEG and dual-DEG architectures. A numerical case study is also reported for specific geometrical dimensions of the buoy and specific wave climate data.
\end{abstract}

\section{INTRODUCTION}

Energy carried by ocean waves is an interesting kind of renewable resource because of its wide and abundant availability (3.7 TW of worldwide usable power [1]) and its high spatial density (an average density of $2-8 \mathrm{~kW} / \mathrm{m}^{2}$ [2], with respect to few hundreds $\mathrm{W} / \mathrm{m}^{2}$ for the solar radiation). Devices that are conceived to scavenge this kind of energy are named Wave Energy Converters (WECs). To date, a quite large number of them has been proposed and tested [2,3].

WEC architectures are based on a combination of two main mechanical sub-systems: 1) a primary interface (buoy, flap, floating cylinders etc.) that is a body that moves under the forces of waves; 2) a Power Take-Off system (PTO), usually employing a combination of hydraulic machinery and electromechanical generators, which converts the mechanical power captured by the primary interface into electricity. To date, current WEC systems are exhibiting a number of technical criticalities that are impeding their practical exploitation: 1) they include a large number of heavy, expensive and corrosion sensitive components; 2) they present complex architectures with high failure probability; 3) traditional PTOs do not adapt easily to the sea environment and do not operate well at the low characteristic frequencies of sea waves $(\sim 0.1 \mathrm{~Hz})$.

Recently, PTO systems based on Dielectric Elastomers (DEs) have been proposed [4-8] for WECs. DEs are highly compliant incompressible polymeric materials that are electrically non-conductive and can be employed to conceive electromechanical transducers. The most well-known DE materials are silicone, acrylic and natural rubbers. DE transducers are made by one or multiple layers of dielectric material coated by compliant electrodes [9]. Their operating principle is that of a deformable electric capacitor, which directly converts the mechanical energy that is spent for the deformation into electricity.

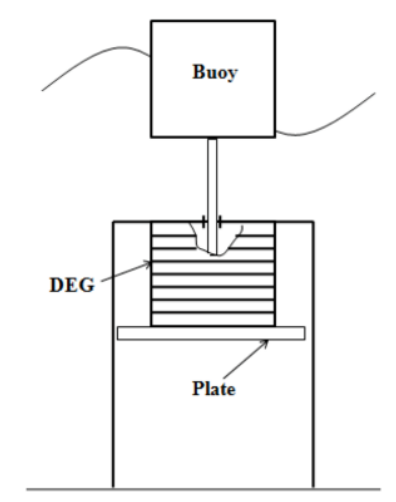

a)

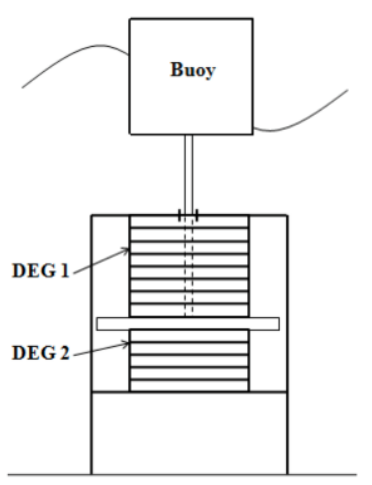

b)

Figure 1. Schematic of Poly-Buoy: a) with single DEG, b) with agonist-antagonist DEG

The employment of DE Generators (DEG) as alternative PTOs for WECs may bring a number advantages, since: 1) they 
exploit lightweight and low-cost materials; 2) their working principle is intrinsically adaptable to the low frequencies of waves; 3) their architecture requires a reduced number of moving parts and, thus, is quite simplified with respect to that of traditional systems.

This paper analyzes the application of DEGs to buoy-based WECs. Buoy-based systems are a rather simple and interesting type of WEC. They belong to a class of deep-water devices, which employ heaving buoys for the primary interface and hydraulic rams or linear electric generators for the PTO system $[3,23,24]$. The considered architecture, herein referred to as Poly-Buoy (see Figure 1), is characterized by using a Stacked DEG (S-DEG) for the PTO system. In Poly-Buoy, cyclical deformation of the S-DEG is induced by a plate (or piston) that is rigidly connected to the heaving buoy. Two layouts are analyzed for the polymeric PTO: the single-DEG architecture (depicted in Figure 1.a); the dual-DEG architecture (depicted in Figure 1.b). The single-DEG layout includes one PTO only, which is constituted by a cylindrical S-DEG having the lower base attached to the moving plate and the upper base attached to a hull that is fixed to the seabed. A similar concept is investigated in the context of EPoSil project, carried out by a German consortium of companies and universities [6]. The Dual-DEG agonist-antagonist [10] layout is obtained by the former one by adding a second identical S-DEG, with the upper base connected to the moving plate and the lower base attached to the fixed hull.

In the paper, the analytical model of the S-DEG and the hydrodynamic analysis of the buoy-based WEC are first introduced. Second, a design approach for the polymeric PTO is proposed, which considers both single-DEG and dual-DEG architectures. Finally, a numerical case study is presented, which uses a commercial silicone rubber (TC-5005 A/B, BJB Enterprises Inc., USA) as reference DE material. Results demonstrate that, for the same power output, the dual-DEG architecture brings to considerable savings in DE material usage as compared to the single-DEG solution.

\section{STACKED DIELECTRIC ELASTOMER GENERATOR}

The Stacked DEG (S-DEG) is constituted by a number of layers of DE membranes coated with compliant electrodes that are piled-up to form a stack. Typically, successive membranes are electrically connected in parallel with alternating polarities of adjacent layers [11]. A scheme of the S-DEG with external electrical circuit is shown in Figure 2.

The multi-layered architecture of S-DEG allows to compactly arrange a large amount of material while guaranteeing homogeneous stretches and electric field within the layers [12].

Stacked generators and actuators are usually operated in compression only [13-15] (i.e. layers are always compressed by external forces). However, bidirectional operation is possible using special designed electrodes based on nano-scale carbon powder as proposed by Kovacs et al. [11]. In the present article, we suppose that the employed S-DEG can operate both in compression and in tension.
This section introduces a mathematical model of the $\mathrm{S}$ DEG which makes it possible to predict (1) the amount of electrical energy that can be converted and (2) the force-stroke response of the generator that is fundamental to assess how the device can interact dynamically with the primary interface (the buoy) of the WEC.

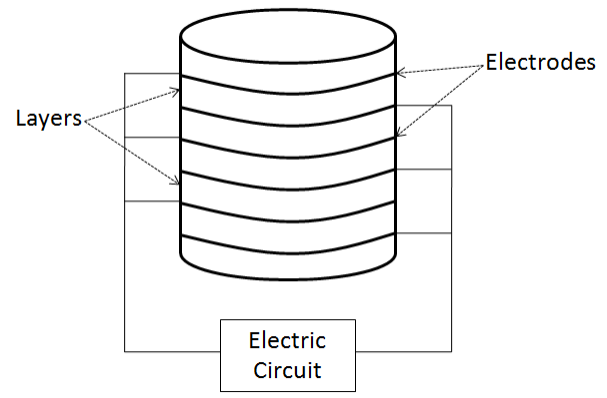

Figure 2. Schematic of the stacked DEG.

A cylindrically shaped S-DEG is considered that, during its operation, is stretched along the longitudinal direction (i.e. along the axis of its cylindrical shape). In term of dimensions, the S-DEG is characterized by the height $h_{0}$ and radius $r_{0}$, which are measured in the undeformed state.

For simplicity, constraining effects at the cylinder bases are neglected so that the deformation state of the $\mathrm{S}-\mathrm{DEG}$ is considered as being uniform and equi-biaxial. Of course, the presence of rigid connection ends at the S-DEG bases is likely to provoke more complex states deformations (with the central layers of the stack undergoing larger surface expansion than the peripheral layers $[13,16])$, but the simplification is deemed sufficient for the purpose of this study.

The coordinate $x$ (see Figure 3 ) identifies the position of the moving plate that is connected to the S-DEG. For $x=0$ the stack is subjected to a longitudinal pre-stretch $\lambda_{p}$.

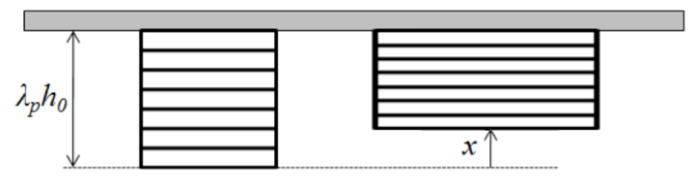

a)

b)

Figure 3. Stacked DEG in reference configuration a) and with a positive displacement $x$ with respect to the reference configuration.

Figure 3 represents the cylindrical stack in the reference undeformed configuration (Figure 3.a) and in a generic deformed configuration (Figure 3.b).

For a given $x$, the principal stretch of the S-DEG in the longitudinal direction reads as

$\lambda_{1}=\lambda=\lambda_{p}-x / h_{0}$.

Besides, due to DE material incompressibility [17], namely

$\lambda_{1} \lambda_{2} \lambda_{3}=1$ 
the principal transversal stretches follow as

$$
\lambda_{2}=\lambda_{3}=1 / \sqrt{\lambda} \text {. }
$$

The force-stroke response of the S-DEG is calculated by means of an energy balance. The state of the generator in any generic configuration is described by two variables: the longitudinal coordinate $x$ and the electric field $E$ acting within the DE layers. The total charge $Q$ on the electrodes or the electric potential difference $V$ between the terminals could be alternatively chosen as electrical state variables.

The energy balance for the S-DEG is expressed in differential form as

$$
d U_{e}+d U_{m}=d W_{e}+d W_{m},
$$

where $U_{e}$ and $U_{m}$ are the electrostatic and elastic potential energies stored in the S-DEG, and $W_{e}$ and $W_{m}$ are the electrical and mechanical works done on the S-DEG by external means.

The electrostatic potential energy is calculated as

$$
U_{e}=\Omega \varepsilon E^{2} / 2,
$$

where $\Omega$ and $\varepsilon$ are the volume and dielectric constant of the employed DE material.

The elastic potential energy depends on the stretch field in the material and can be written as

$$
U_{m}=\Omega \Psi(x),
$$

where $\Psi$ is the strain-energy function (or Helmholtz function) of the considered DE material. In this article, a Gent hyperelastic form is used for $\Psi$ [17], namely

$$
\Psi=-a \log \left(\frac{\mathrm{I}_{m}-\lambda_{1}{ }^{2}-\lambda_{2}{ }^{2}-\lambda_{3}^{2}}{\mathrm{I}_{m}-3}\right),
$$

where $I_{m}$ and $a$ are the material constitutive parameters.

The infinitesimal electrical work done by the external electrical circuit connected to the S-DEG is calculated as

$d W_{e}=V d Q$,

that can be expressed as a function of $x$ and $E$ as

$$
d W_{e}=\Omega \varepsilon E^{2} d E+\frac{\Omega \varepsilon E^{2}}{\lambda_{p} h_{0}-x} d x
$$

The infinitesimal mechanical work performed by the external force applied to the DE stack is calculated as

$d W_{m}=-F_{D E G} d x$,

where $F_{D E G}$ is the force exerted by the S-DEG on the external environment.

In balance (4) and in the successive equations, the following aspects have been neglected: 1) visco-elastic losses of the polymeric material; 2) inertial and gravitational forces acting on the polymer; 3) leakage currents through the dielectric medium; 4) electric losses due to the conditioning circuit and to charge dispersion from the electrodes.

Combining equation (4) with equations (5), (6), (9) and (10) yields the force provided by the S-DEG, that is

$F_{D E G}=F_{D E G, e}+F_{D E G, m}$,

where $F_{D E G, e}$ and $F_{D E G, m}$ are the following electrostatic and mechanical contributions

$$
F_{D E G, e}=\frac{\varepsilon \Omega E^{2}}{\lambda_{p} h_{0}-x}, F_{D E G, m}=-\Omega \frac{d \Psi}{d x} .
$$

Besides, based on the same equations, the amount of electrical energy produced/spent by the S-DEG for an infinitesimal deformation $d x$ is

$d U_{e}-d W_{e}=-\frac{\varepsilon \Omega E^{2}}{\lambda_{p} h_{0}-x} d x$.

From equation (13), it is clear that the S-DEG generates electrical energy only when $E \neq 0$ and $d x<0$; that is, when the $S$-DEG capacitance decreases.

\section{OPERATION CONSTRAINTS}

S-DEG design and operation is subjected to restrictions determined by the following set of failure conditions [18]:

Electric Break-down: the maximum value of electric field in the dielectric membranes must be smaller than the dielectric strength, $E_{B D}$, of the dielectric material; namely $E \leq E_{B D}$.

Mechanical Rupture: the maximum reachable stretch is set to a limit value, $\lambda_{u}$. This condition must apply for $\lambda_{1}, \lambda_{2}$ and $\lambda_{3}$. This results in a constraint on the longitudinal stretch, namely

$\lambda_{u}^{-2} \leq \lambda \leq \lambda_{u}$.

Compression buckling: when the S-DEG is compressed, it may be subjected to instability (namely, buckling). A criterion that can be used to approximate the arising of buckling in SDEGs is provided by Haringx formula [19] [20], which derives from the extension of Euler's theory to the study of the buckling of rubber blocks. Haringx formula estimates the first critical load as

$$
F_{c r}=\frac{G A}{2}\left(\sqrt{1+\frac{4 P_{E}}{G A}}-1\right),
$$

where $A$ is the actual cross section of the rubber block, $G$ is the shear modulus of the material and $P_{E}$ is Euler's critical load. Considering a cylindrical beam with guided ends, Euler's load follows as

$P_{E}=\pi^{3} E r^{4} / 4 h^{2}$,

where $r$ and $h$ are the actual radius and height of the rubber block, while $E$ is Young's elasticity modulus. For hyperelastic materials having a strain-energy function given by equation (7), 
$G$ and $E$ are not constant and depend on the state of deformation that is described by $\lambda$. Here, $G$ and $E$ are evaluated from (7) at $\lambda=1$, which yields

$$
\begin{aligned}
& G=2 a /\left(I_{m}-3\right), \\
& E=3 G .
\end{aligned}
$$

Notice that this is a strong assumption, which may lead to a very approximated estimation of the buckling load. Nonetheless, the approximation is considered sufficient for the purpose of this study.

The condition described by equations (15)-(16) is used to determine a minimum allowed value for the longitudinal stretch, $\lambda_{c r}$, that prevents the S-DEG from buckling. In order to calculate $\lambda_{c r}, F_{c r}$ in (15) is replaced by the magnitude of the mechanical part of the S-DEG force (that is, $F_{D E G, m}$ given by equation (12)), which yields the following equation in the single variable $\lambda$

$$
-\frac{\lambda}{G} \frac{\partial \psi}{\partial \lambda}=\frac{1}{2}\left[\sqrt{1+\frac{3 \pi^{2}}{\lambda^{3}}\left(\frac{r_{0}}{h_{0}}\right)^{2}}-1\right] .
$$

Solution of (19) provides the critical buckling stretch $\lambda_{c r}$.

For a given geometry of the S-DEG, the failure conditions described above restrict the operating range of the generator. In particular, the combination of mechanical rupture and buckling establishes that the minimum allowed longitudinal stretch is

$\lambda_{\min }=\max \left\{\lambda_{u}^{-2} ; \lambda_{c r}\right\}$.

Overall, the considered set of limitations provides an upper bound to the amount of energy that the S-DEG can convert in a cycle. Specifically, the maximum energy per unit volume can be calculated from (13) by imposing $E=E_{B D}$, thereby obtaining

$E_{\max }=\varepsilon E_{B D}^{2} \log \left(\lambda_{u} / \lambda_{\min }\right)$.

\section{THE HEAVING BUOY WEC}

In this section, a hydrodynamic model for a heaving buoy WEC is described and possible control strategies for an ideal PTO are examined. An analysis of a particular numerical case is also provided to set a reference for the design of the S-DEG PTO that is conducted in the subsequent section.

The buoy WEC considered in this article is a floating vertical cylinder that oscillates along its axial direction under wave forces and generates electrical energy by means of a proper PTO system.

In order to gain an accurate understanding of the dynamics of a floater driven by sea waves, the usage of sophisticated tools (like CFD codes) would be required, but this implies complex and time-consuming calculations. A preliminary but sufficiently accurate estimation of a WEC dynamics can be obtained using potential flow and linear water wave theory [24]. Under these hypotheses, the general equation of motion of a heaving buoy WEC takes the form below:

$$
\left(M+M_{\infty}\right) \ddot{x}+\int_{0}^{t} K(t-\tau) \dot{x}(\tau) d \tau+k_{b} \dot{x}=F_{\text {exc }}(t)+F_{\text {PTO }}(t),
$$

where

- $\quad M$ is the mass of the floater and $M_{\infty}$ is the added mass at infinite frequency;

- $\quad k_{b}=\rho g S$ is the hydrostatic stiffness coefficient with $\rho$ being the water density and $S$ being the buoy transversal section;

- the integral term in equation (22) is a convolution integral which represents the effect of the radiated waves generated by the buoy motion;

- $F_{\text {exc }}(t)$ is the wave excitation force;

- $F_{\text {PTO }}(t)$ is the force supplied by the PTO.

In this study, regular waves are considered, so that the exciting force takes the form

$F_{e x c}(t)=\Gamma \sin \omega t$.

Note that using regular monochromatic waves leads to an overestimation of the WEC power output [25]. Nonetheless, this hypothesis allows the simplification of the mathematical analysis and is deemed sufficiently adequate for a preliminary evaluation of the WEC performance.

Although the hydrodynamics in equation (22) are linear, the PTO response $F_{\text {PTO }}$ can be, in general, non linear. In this

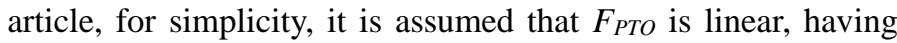
the form

$$
F_{P T O}=-\left(k_{P T O} x+B_{P T O} \dot{x}\right),
$$

where $k_{P T O}$ and $B_{\text {PTO }}$ are the stiffness and the damping provided by the PTO. This is a rather common assumption in the study of WECs and makes it possible to solve analytically the dynamics of the floater using a frequency-domain approach instead of a time-domain analysis [25].

Under the hypothesis of monochromatic waves and linear PTO (that is, due to equations (23) and (24)), the convolution term in (22) simplifies, resulting in an added-mass term plus a radiation damping effect [3]:

$$
\int_{0}^{t} \dot{x}(\tau) K(t-\tau) d \tau \approx\left(M_{\text {add }}(\omega)-M_{\infty}\right) \ddot{x}+B_{r}(\omega) \dot{x},
$$

where $M_{\text {add }}(\omega)$ and $B_{r}(\omega)$ are the added mass and the radiation damping coefficients, which depend on the excitation frequency.

The quantities $M_{\text {add }}(\omega), M_{\infty}, B_{r}(\omega)$ and $\Gamma$ are obtained using a boundary element code (WAMIT [26]) for different choices of the incident wave frequency.

Based on the above, the average power that can be extracted by the WEC in a generic monochromatic sea state results as [8]

$$
P=\frac{B_{P T O}}{2} \int_{T} \dot{x}^{2} d t=0.5 B_{P T O} \omega^{2} \Gamma^{2} /\left[\left(k-I \omega^{2}\right)^{2}+B^{2} \omega^{2}\right],
$$

where $k$ and $B$ are defined as 
$k=k_{b}+k_{\text {PTO }}$,

$B=B_{r}+B_{P T O}$.

By adjusting the parameters $k_{P T O}$ and $B_{P T O}$ to the different sea state conditions, the PTO can maximize the captured energy.

Among the possible strategies, two of the most representative types of controllers are considered: linear suboptimal control and linear optimal control. For both the controllers the following motion constraint on the oscillation amplitude of the WEC is introduced

$|x| \leq X_{\max }$.

Imposition of this constraint is advisable since: 1) large oscillations (with resulting large velocities) provoke vortexshedding phenomena, that are not accounted in the model and lead to severe energetic losses; 2) the oscillation of the buoy is intrinsically limited by the non-linear hydrodynamics that are not included in the considered model.

\section{Linear suboptimal control}

The reactive term governed by the coefficient $k_{P T O}$ in equation (24) is very difficult to implement in practice [28]. Thus, the simplest mathematically linear PTO provides a controllable damping effect only (that is known under the name of "linear suboptimal control"). Assuming $k_{P T O}=0$, maximization of WEC power output only requires the tunable parameter $B_{P T O}$ to be adjusted according to the wave excitation frequency.

The analytical optimum value for $B_{\text {PTO }}$ is [8]

$B_{P T O}=\left\{\begin{array}{c}\sqrt{B_{r}^{2}+\frac{\left(k-\left(M+M_{a d d}\right) \omega^{2}\right)^{2}}{\omega^{2}}} \text { if } \bar{x} \leq X_{\max } \\ \frac{1}{\omega} \sqrt{\frac{\Gamma^{2}}{X_{\max }^{2}}-\left(k-\left(M+M_{a d d}\right) \omega^{2}\right)^{2}}-B_{r} \text { if } \bar{x}>X_{\max }\end{array}\right.$

where $\quad \bar{x}, \quad \bar{x}=\Gamma / \sqrt{\left(k-\left(M+M_{\text {add }}\right) \omega^{2}\right)^{2}+B^{2} \omega^{2}}, \quad$ is the unbounded oscillation amplitude of the buoy.

\section{Linear optimal control}

In the best case, both $k_{P T O}$ and $B_{P T O}$ can be tuned for different sea states. In this circumstance, the maximum power output of the WEC is achieved when [8]

$k_{P T O}=\left(M+M_{a d d}\right) \omega^{2}-k_{b}$,

$B_{P T O}=\left\{\begin{array}{c}B_{r} \text { if } \Gamma /\left(2 \omega B_{r}\right) \leq X_{\max } \\ \Gamma /\left(\omega X_{\max }\right)-B_{r} \text { if } \Gamma /\left(2 \omega B_{r}\right)>X_{\max }\end{array}\right.$.

\section{Analysis of a reference case}

In this section, the typical characteristic responses of a generic PTO that are required for the implementation of linear optimal and suboptimal controllers are presented via a specific case study. As regards reference sea states, the wave data provided in [27] are considered. The twenty most probable sea conditions, among those reported in that paper, are used as target for the present analysis. Specifically, the reference polychromatic sea states are converted into regular monochromatic sea conditions using the procedure described in [8]. Each Sea State (SS) is identified by two wave variables: period $T$ and height, $H$, that are shown in Table 1 .

As regards the primary interface, the physical dimensions, properties and displacement limit assumed for the buoy are shown in Table 2.

\begin{tabular}{|r|r|r|r|r|r|}
\hline SS & $\boldsymbol{T}[\mathbf{s}]$ & $\boldsymbol{H}[\mathbf{m}]$ & $\#$ & $\boldsymbol{T}[\mathbf{s}]$ & $\boldsymbol{H}[\mathbf{m}]$ \\
\hline 1 & 12.4 & 1.2 & 11 & 11.7 & 1.2 \\
\hline 2 & 8.7 & 2.5 & 12 & 7.1 & 1.2 \\
\hline 3 & 9.9 & 3.4 & 13 & 8.8 & 2.4 \\
\hline 4 & 10.5 & 2.4 & 14 & 8.4 & 2.3 \\
\hline 5 & 8.4 & 2.3 & 15 & 7.2 & 1.5 \\
\hline 6 & 10.0 & 3.6 & 16 & 8.9 & 1.6 \\
\hline 7 & 10.0 & 3.4 & 17 & 7.2 & 1.5 \\
\hline 8 & 10.5 & 2.2 & 18 & 9.0 & 1.3 \\
\hline 9 & 11.9 & 3.1 & 19 & 7.2 & 1.3 \\
\hline 10 & 11.5 & 3.1 & 20 & 8.9 & 1.4 \\
\hline
\end{tabular}

Table 1. Wave period and height for the 20 reference sea states.

\begin{tabular}{|c|c|}
\hline Diameter & $10 \mathrm{~m}$ \\
\hline Draft & $9.4 \mathrm{~m}$ \\
\hline Mass & $738 \times 10^{3} \mathrm{~kg}$ \\
\hline Displacement limits $\left(X_{\max }\right)$ & $\pm 8 \mathrm{~m}$ \\
\hline
\end{tabular}

Table 2 . Properties of the reference buoy.

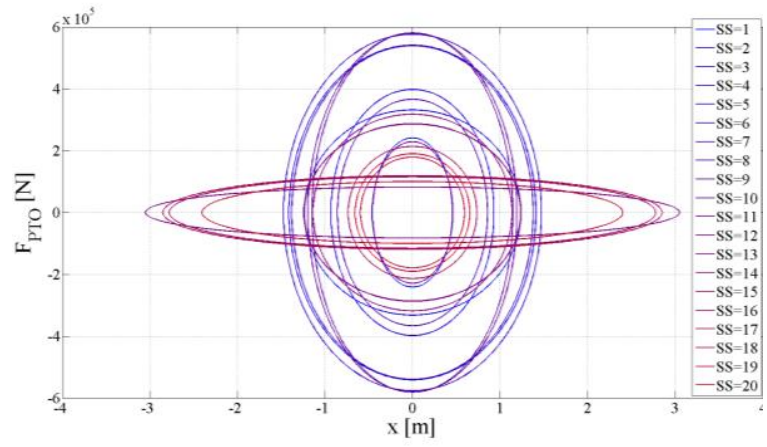

Figure 4. Elliptical trajectories for the linear suboptimal case.

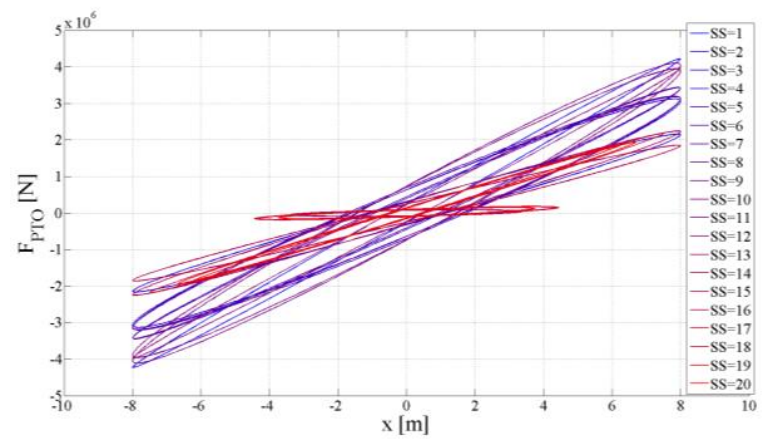

Figure 5. Elliptical trajectories for linear optimal case. 
The resulting force-displacement responses are shown in Figures 4 and 5 for suboptimal and optimal linear controllers, respectively. As shown, in the plane $x-F_{\text {PTO }}$, trajectories determined by equation (24) represent ellipses (each ellipses being for a specific sea state). This type of graphical representation is fundamental for the design of DEG PTOs, for which the force characteristic is naturally expressed as a function of position.

Specifically, Figure 4 highlights that the response of linear suboptimal control is characterized by ellipses with principal directions aligned to the horizontal and vertical axes, and requires a PTO featuring a peak power of $267 \mathrm{~kW}$ and a maximum stroke of about $\pm 3 \mathrm{~m}$.

Besides, Figure 5 shows that the optimal controller is characterized by elliptical trajectories whose principal directions do not coincide with the coordinate axes (this is due to the stiffness compensation introduced by $k_{P T O}$ ), and requires a PTO featuring a peak power of $1.75 \mathrm{MW}$ and a maximum stroke of $\pm 8 \mathrm{~m}$ (that equals the imposed displacement limit).

\section{CASE STUDY OF PTO DESIGN}

This section is dedicated to a case study in which a cylindrical S-DEG is dimensioned to be used as PTO for the Poly-Buoy system with the specific properties and sea state conditions described in the previous section. In particular, the proposed design procedure aims at identifying the properties of the S-DEG PTO that enable the implementation of the optimal and suboptimal controllers introduced before.

\section{Dielectric elastomer properties}

As regards the $\mathrm{S}-\mathrm{DEG}$ material, a reference silicone elastomer (TC-5005 A/B-C by BJB Enterprises Inc., USA) is considered whose properties are summarized in Table 1 [21]. Based on (21), the related maximum convertible energy density is $1.69 \times 10^{6} \mathrm{~J} / \mathrm{m}^{3}$. In the following, it will be shown that this theoretical value cannot be reached when using S-DEGs as PTO for Poly-Buoys, specifically if one seeks to maximize the overall system power extraction.

\begin{tabular}{|c|c|c|}
\hline  & $E_{B D}$ & $100 \mathrm{MV} / \mathrm{m}$ \\
\hline$\frac{0}{x}$ & $\varepsilon_{r}$ & 4.6 \\
\hline \multirow{3}{*}{  } & $a$ & $8.17 \times 10^{5} \mathrm{~Pa}$ \\
\hline & $I_{m}$ & 72.58 \\
\hline & $\lambda_{u}$ & 4 \\
\hline
\end{tabular}

Table 3. Electro-mechanical properties of TC-5005 A/B.

\section{S-DEG dimensioning}

To implement either the suboptimal or the optimal control in the considered Poly-Buoy system, the S-DEG to be designed is required to have an operational space in the $x-F_{\text {PTO }}$ plane that must envelope all the ellipses (for all the considered sea states) reported either in Figure 4 or in Figure 5.

Based on equations (11) and (12), for the single-DEG architecture, the PTO force reads as

$F_{P T O}=F_{D E G}(x, E)$.

This constrains the force-displacement response of the PTO to lie anywhere within two curves: a lower one obtained for $E=0$ and an upper one obtained for $E=E_{B D}$.

For the double-DEG architecture, the PTO force reads as

$F_{P T O}=F_{D E G}\left(x, E_{\mathrm{up}}\right)-F_{D E G}\left(-x, E_{\mathrm{down}}\right)$,

with $E_{\text {up }}$ and $E_{\text {down }}$ respectively indicating the electric fields to be controlled in the upper and lower parts of the agonistantagonist generator. In this case, the force-displacement response of the PTO is constrained to lie anywhere within the two curves that are obtained for the reciprocal activation (namely, either $\left[E_{\mathrm{up}}=E_{B D} \& E_{\text {down }}=0\right]$ or $\left[E_{\text {up }}=0 \& E_{\text {down }}=\right.$ $\left.\left.E_{B D}\right]\right)$ of the upper and lower parts of the generator.

In the following, different S-DEG designs are reported which exhibit operational spaces that entirely include the set of control trajectories depicted in Figure 4 and Figure 5 (that is, all the points in the $x-F_{\text {PTO }}$ plane that are required to be reached for the implementation of the suboptimal and optimal controllers). For each case, the considered design variables are: the overall DE material volume, $\Omega$; the undeformed height of the stack, $h_{0}$; the longitudinal pre-stretch, $\lambda_{p}$. Reported designs are for these variables optimized in order to minimize the amount of DE material employed while respecting the operational constraints that are required to prevent S-DEG failure (namely, dielectric strength, mechanical rupture and buckling).

Specifically, three variants are distinguished and analyzed in all the various combinations:

1) Linear suboptimal or optimal control;

2) Single-DEG or Dual-DEG PTO;

3) Presence or absence of an additional passive mechanical component (namely, a mechanical spring) that is placed in parallel to the S-DEG.

The addition of a mechanical spring with stiffness $k_{s}$ (that can be positive or negative) is considered to provide a means to shift the natural resonance frequency of the overall system. When included, the value of $k_{s}$ is an additional parameter to be optimized.

Table 4 and Table 5 provide the results for the linear suboptimal and optimal control, respectively. Tables report the optimal geometric parameters for the S-DEGs together with the related force-displacement curves. As shown, for all cases, the operational space of the S-DEG fully envelopes the control ellipses. When the mechanical spring is present, the curves representing the S-DEG response are calculated including the force contribution provided by $k_{s}$. The value for the converted energy density of the S-DEG is calculated with reference to the peak energy captured by the WEC in a wave period. 


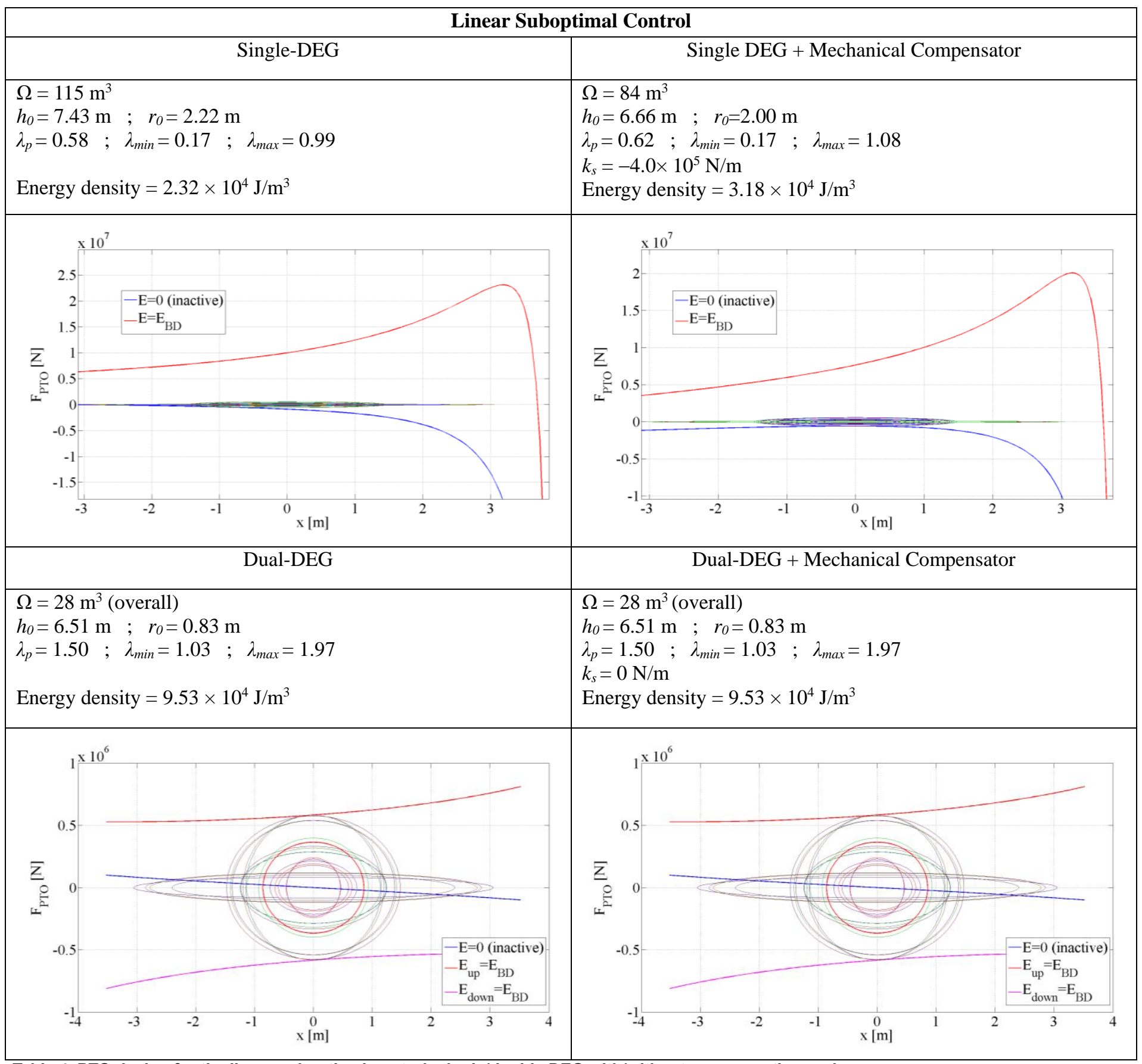

Table 4. PTO design for the linear suboptimal control: single/double DEG with/without compensation spring. 




Table 5. PTO design for the linear optimal control: single/double DEG with/without compensation spring.

The results in Table 4 and Table 5 show that:

- In most of the considered cases, the optimal value of $k_{s}$ is different from zero and is negative. The effect of the external passive spring-like component is in general beneficial, allowing to save a large amount of DE material. Nonetheless, the introduction of a mechanical spring may be technically difficult since: 1) it requires further metallic material to be added for the spring; 2) springs with negative stiffness may be difficult to realize.

- The single-DEG layout requires larger quantities of DE material than the dual-DEG solution. Indeed, to cover the whole operating space identified by the elliptical trajectories, the S-DEG must be able to provide negative forces. That is, the S-DEG must be compressed for large part of its operation. Since the operation of the DEG in compression is strongly affected by the buckling limit, this results in an increase in the amount of DE material needed.

- In order to envelop the elliptical trajectories in the $x-F_{P T O}$ plane, the electric field controller is required to activate the S-DEG also when its capacitance is increasing (which requires the S-DEG to be used also as an actuator and not as a generator only).

- The assessed operational energy density for the reference silicone is significantly lower than the maximal theoretical 
value given by equation (21). This demonstrates that it is rather difficult to fit the optimal control curves of a PolyBuoy with a S-DEG; that is, the maximization of the energy extraction of the overall Poly-Buoy system implies a nonoptimal exploitation of the DE material.

- Although the linear optimal control allows to obtain larger power outputs than the linear suboptimal control, its implementation requires a larger amounts of DE material. In particular, the implementation of a linear control strategy using a single-DEG layout requires unreasonable amounts of DE material, leading to unfeasible technical solutions. In practice, a compromise has to be found between the cost of DE material and the amount of electricity produced.

\section{CONCLUSIONS}

In this article, a novel concept of Wave Energy Converter (WEC) with Dielectric Elastomer (DE) Power Take-Off (PTO) system has been investigated. The considered device, named Poly-Buoy, has a heaving buoy as primary mechanical interface and a cylindrical Stacked DE Generator (S-DEG) as PTO.

An analytical model of the S-DEG has been proposed to describe the force response of the device and to evaluate the energy that can be converted. The limits of operation for the SDEG are determined by imposing a set of failure conditions that includes electric break-down, mechanical rupture and compression buckling.

A hydrodynamic model of the buoy-type WEC has been described, which is based on linear potential theory.

A numerical case study has been presented considering a buoy with $10 \mathrm{~m}$ diameter and $9.4 \mathrm{~m}$ draft, and operating in a reference wave climate. Different cases have been studied:

- Linear optimal and suboptimal control strategies, that are a standard in WEC control theory;

- Single- and dual-DEG agonist-antagonist architectures;

- S-DEG with mechanical compensation obtained by means of a passive negative spring.

Numerical calculations showed that, under the assumed simplifications, the amount of volume necessary to achieve the mentioned control laws is at least $28 \mathrm{~m}^{3}$ for the linear suboptimal control (which makes it possible to capture an average power of $267 \mathrm{~kW}$ for the best sea state) and $174 \mathrm{~m}^{3}$ for the linear optimal case (which makes it possible to capture an average power of $1.75 \mathrm{MW}$ for the best sea state).

The use of an agonist-antagonist dual-DEG architecture significantly reduces the amount of DE material needed.

It should be remarked that the design of a complex system, like the Poly-Buoy, requires to find a compromise between different factors, which depend on both the dynamics of the floating WEC and on the operation of the DEG PTO. Maximization of the captured energy by the Poly-Buoy leads to a sub-optimal utilization of the DE material (that is, at energy densities that are very far from the maximum theoretical value). For a more in-depth study of Poly-Buoy systems, technoeconomic considerations should be included in the analysis.

\section{ACKNOWLEDGMENTS}

The work presented in this paper is developed in the context of the project PolyWEC (www.polywec.org), a FP7 FET-Energy project. The research leading to these results has received funding from the European Union Seventh Framework Programme [FP7/2007-2013] under grant agreement $\mathrm{n}^{\circ} 309139$.

The authors sincerely thank dr. David Forehand (Edinburgh University) for kindly providing the WAMIT hydrodynamic parameters of the described WEC.

\section{REFERENCES}

[1] Mork, G., Barstow, S., Kabuth, A., \& Pontes, M. T. (2010). Assessing the global wave energy potential. In ASME 2010 29th International Conference on Ocean, Offshore and Arctic Engineering. American Society of Mechanical Engineers.

[2] Falnes, J. (2007). A review of wave-energy extraction. Marine Structures, 20(4), 185-201.

[3] Falcão, A. (2010). Wave energy utilization: A review of the technologies. Renewable and sustainable energy reviews, 14(3), 899-918.

[4] Chiba, S., Waki, M., Kornbluh, R., \& Pelrine, R. (2008). Innovative power generators for energy harvesting using electroactive polymer artificial muscles. In The 15th International Symposium on: Smart Structures and Materials \& Nondestructive Evaluation and Health Monitoring. International Society for Optics and Photonics.

[5] Jean, P., Wattez, A., Ardoise, G., Melis, C., Van Kessel, R., Fourmon, A., \& Queau, J. P. (2012). Standing wave tube electro active polymer wave energy converter. In: SPIE Smart Structures and Materials, pp. 83400C83400C.

[6] Scherber, B., Grauer, M., \& Köllnberger, A. (2013). Electroactive polymers for gaining sea power. In SPIE Smart Structures and Materials Nondestructive Evaluation and Health Monitoring. International Society for Optics and Photonics.

[7] Vertechy, R., Fontana, M., Rosati Papini, G., \& Bergamasco, M. (2013). Oscillating-water-column waveenergy-converter based on dielectric elastomer generator. In SPIE Smart Structures and Materials \& Nondestructive Evaluation and Health Monitoring. International Society for Optics and Photonics.

[8] Moretti, G., Forehand, D., Fontana, M., Vertechy, R., \& Ingram, D. (2014). Modeling of an Oscillating Wave Surge with Dielectric Elastomer Power Take-Off. In ASME 2010 33rd International Conference on Ocean, Offshore and Arctic Engineering. American Society of Mechanical Engineers.

[9] Carpi, F., De Rossi, D., Kornbluh, R., Pelrine, R. E., \& Sommer-Larsen, P. (2011). Dielectric elastomers as electromechanical transducers: Fundamentals, materials, devices, models and applications of an emerging electroactive polymer technology. Elsevier. 
[10] Fontana, M., Moretti, G., Lenzo, B., Vertechy, R. (2014). Loading system mechanism for dielectric elastomer generators with equi-biaxial state of deformation. In SPIE Smart Structures and Materials + Nondestructive Evaluation and Health Monitoring. International Society for Optics and Photonics.

[11] Kovacs, G., Düring, L., Michel, S., \& Terrasi, G. (2009). Stacked dielectric elastomer actuator for tensile force transmission. Sensors and Actuators A: Physical, 155(2), 299-307.

[12] Niu, X., Leo, R., Chen, D., Hu, W., \& Pei, Q. (2013). Multilayer stack actuator made from new prestrain-free dielectric elastomers. In SPIE Smart Structures and Materials + Nondestructive Evaluation and Health Monitoring. International Society for Optics and Photonics.

[13] Anderson, I. A., Rosset, S., McKay, T., \& Shea, H. (2014). Stack design for portable artificial muscle generators: is it dangerous to be short and fat?. In SPIE Smart Structures and Materials + Nondestructive Evaluation and Health Monitoring. International Society for Optics and Photonics.

[14] Chuc, N. H., Vuong, N. H. L., Kim, D. S., Moon, H. P., Koo, J. C., Lee, Y. K., Nam, J. D., \& Choi, H. R. (2011). Fabrication and control of rectilinear artificial muscle actuator. IEEE/ASME Transactions on Mechatronics, 16(1), 167-176.

[15] Price, A. D. (2013). Evolutionary algorithms for the multi-objective optimization of stacked dielectric elastomer actuators. In SPIE Smart Structures and Materials + Nondestructive Evaluation and Health Monitoring. International Society for Optics and Photonics.

[16] Kaal, W., \& Herold, S. (2013). Numerical investigations on dielectric stack actuators with perforated electrodes. Smart Materials and Structures, 22(10), 104016.

[17] Holzapfel, G. A. (2000). Nonlinear solid mechanics: a continuum approach for engineering. West Sussex, England: John Wiley \& Sons, Ltd.

[18] Jin Adrian Koh, S., Keplinger, C., Li, T., Bauer, S., \& Suo, Z. (2011). Dielectric elastomer generators: How much energy can be converted? IEEE/ASME Transactions on Mechatronics, 16(1), 33-41.

[19] Haringx, J. A. (1942). On the buckling and lateral rigidity of helical springs. Proc. Konink. Ned. Akad. Wet, 45(533), 142.
[20] Bazant, Z. P. (2003). Shear buckling of sandwich, fiber composite and lattice columns, bearings, and helical springs: Paradox resolved. Journal of applied mechanics, 70(1), 75-83.

[21] Vertechy, R., Frisoli, A., Bergamasco, M., Carpi, F., Frediani, G., \& De Rossi, D. (2012). Modeling and experimental validation of buckling dielectric elastomer actuators. Smart Materials and Structures, 21(9), 094005.

[22] Pelrine, R., Kornbluh, R. D., Eckerle, J., Jeuck, P., Oh, S., Pei, Q., \& Stanford, S. (2001). Dielectric elastomers: Generator mode fundamentals and applications. In SPIE's 8th Annual International Symposium on Smart Structures and Materials (pp. 148-156). International Society for Optics and Photonics.

[23] Waters, R., Stålberg, M., Danielsson, O., Svensson, O., Gustafsson, S., Strömstedt, E., Eriksson, M., Sundberg, J., \& Leijon, M. (2007). Experimental results from sea trials of an offshore wave energy system. Applied Physics Letters, 90(3), 034105.

[24] Babarit, A., Hals, J., Muliawan, M. J., Kurniawan, A., Moan, T., \& Krokstad, J. (2012). Numerical benchmarking study of a selection of wave energy converters. Renewable Energy, 41, 44-63.

[25] De Backer, G., Vantorre, M., Beels, C., De Rouck, J., \& Frigaard, P. (2009). Performance of closely spaced point absorbers with constrained floater motion. In Proceedings of the 8th European Wave and Tidal Energy Conference (pp. 806-817).

[26] WAMIT User Manual Version 7; WAMIT INC., Chestnut Hill, MA (USA); 2014.

[27] Thorpe, T. W. (1999). A brief review of wave energy. (pp. 14) London, UK: Harwell Laboratory, Energy Technology Support Unit.

[28] Folley, M., Whittaker, T., \& Osterried, M. (2004). The oscillating wave surge converter. In Fourteenth International Offshore and Polar Engineering Conference, Toulon, France.

[29] Hals, J., Falnes, J., \& Moan, T. (2011). Constrained optimal control of a heaving buoy wave-energy converter. Journal of Offshore Mechanics and Arctic Engineering, 133(1), 011401.

[30] Falnes, J. (2002). Ocean waves and oscillating systems. Cambridge University Press. 